\title{
INICIAÇÃO CIENTÍFICA COM ALUNOS DO ENSINO FUNDAMENTAL EM ALEGRE- ES: RESULTADOS PARCIAIS DO USO DE PLANTAS MEDICINAIS
}

\author{
Gean Carlos da Silva Abreu ${ }^{1}$ \\ Antônio João Viana Batista ${ }^{2}$ \\ Gean Gimenez ${ }^{3}$ \\ Marroni de Alcântara Barbosa ${ }^{4}$ \\ Amanda Ribeiro Braga ${ }^{5}$ \\ Mauricio Assad ${ }^{6}$ \\ Maria Júlia Alledi de Campos ${ }^{7}$ \\ Mirelle Baptista Jordain ${ }^{8}$ \\ Rudison da Silva Florêncio ${ }^{9}$ \\ Lenir Cardoso Porfirio ${ }^{10}$
}

Resumo: Este artigo apresenta resultados preliminares do trabalho de pesquisa junto a estudantes da EEEFM Aristeu Aguiar, Alegre-Espírito Santo. No projeto são desenvolvidas atividades voltadas a jovens interessados em aprender a preparar domissanitários que tem como base, plantas medicinais cultivadas na horta de plantas medicinais da escola. A importância do método de observação científica é destaque nas atividades dos estudantes, que incluem entre outras: manutenção da horta identificação das espécies; preparo de tinturas e extratos de plantas medicinais usadas para formulação de produtos a serem utilizados como alternativas a problemas do dia a dia da escola, como a desinfecção de ambientes de uso comum no cotidiano dos estudantes. Os bolsistas prepararam, a partir de extrato de alecrim (Rosmarinus officinalis), uma loção que foi, por opção dos alunos, utilizada em teste de controle do bicho mineiro (Leucoptera coffeella) do café (Coffea sp.), com iniciativa observacional dos alunos, o que resultou nos resultados preliminares para a preparação deste artigo.

Palavras-chave: Fitodomissanitários; Pragas; Observação científica; Rosmarinus officinalis.

\footnotetext{
${ }^{1}$ Ensino fundamental/EEEFM Aristeu Aguiar, Brasil. E-mail: gean.s.abreu@gmail.com.

2 Ensino fundamental/EEEFM Aristeu Aguiar, Brasil. E-mail: antonio.jvb@gmail.com.

3 Ensino fundamental/EEEFM Aristeu Aguiar, Brasil. E-mail: gean.gimenes@gmail.com.

${ }^{4}$ Ensino fundamental/EEEFM Aristeu Aguiar, Brasil. E-mail: marroni.ab@gmail.com.

${ }^{5}$ Ensino fundamental/EEEFM Aristeu Aguiar, Brasil. E-mail: amanda.rbraga@gmail.com.

${ }^{6}$ Ensino fundamental/EEEFM Aristeu Aguiar, Brasil. E-mail: assad.mau@gmail.com.

${ }^{7}$ Ensino fundamental/EEEFM Aristeu Aguiar, Brasil. E-mail: alledi.mjulia@gmail.com.

${ }^{8}$ Medicina Veterinária/Universidade Federal do Espirito Santo, Brasil. E-mail: mirelle_bj@gmail.com.

${ }^{9}$ Medicina Veterinária/Universidade Federal do Espirito Santo, Brasil. E-mail: rudi.florencio@gmail.com.

10 Medicina Veterinária/Universidade Federal do Espirito Santo, Brasil. E-mail: lenircp52@gmail.com.
} 